\title{
Gravitational waves from an accreting neutron star with a magnetic mountain
}

\author{
D. J. B. Payne*, A. Melatos* and E. S. Phinney ${ }^{\dagger}$ \\ ${ }^{*}$ School of Physics, University of Melbourne, Parkville, VIC, 3010, Australia \\ ${ }^{\dagger}$ Theoretical Astrophysics, Mail Code 130-33, California Institute of Technology, Pasadena, CA \\ 91125, USA
}

\begin{abstract}
We calculate the amplitude of gravitational waves from a neutron star accreting symmetrically at its magnetic poles. The magnetic field, which is compressed into an equatorial belt during accretion, confines accreted matter in a mountain at the magnetic pole, producing gravitational waves. We compute hydromagnetic equilibria and the corresponding quadrupole moment as a function of the accreted mass, $M_{\mathrm{a}}$, finding the polarization- and orientation- averaged wave strain at Earth to be $h_{c}=6.3 \times 10^{-25}\left(M_{\mathrm{a}} / 10^{-5} M_{\odot}\right)(f / 0.6 \mathrm{kHz})^{2}(\mathrm{~d} / 1 \mathrm{kpc})^{-1}$ for a range of conditions, where $f$ is the wave frequency and $d$ is the distance to the source. This is $\sim 10^{2}$ times greater than previous estimates, which failed to treat the mass-flux distribution self-consistently with respect to flux-freezing.
\end{abstract}

\section{INTRODUCTION}

The direct observation of kilohertz gravitational waves with kilometer-baseline interferometers is presently a realistic goal. Possible transient sources of these waves include supernovae and coalescing neutron star binaries. For periodic sources of known frequency $f$, coherent integration over a time interval $\tau$, can improve the sensitivity by $(f \tau)^{1 / 2}[1]$. Neutron stars can act as periodic sources if their axisymmetry is broken. There are several possible mechanisms in the literature which can deform the star, e.g. thermally [2], or induce it to precess. The dipole magnetic field of an isolated neutron star induces deformations with mass ellipticity $\varepsilon \leq 10^{-9}$, too small to be detected by current interferometers [3].

We explore here a mechanism, known as magnetic burial, which can produce deformations with $\varepsilon \sim 10^{-7}$. In this mechanism, material spreads from the magnetic pole, dragging with it the magnetic field, leaving a compressed band of field at the equator and a reduced magnetic dipole moment $\mu[4,5]$. The equatorial field supports a mountain at the magnetic pole, which generates gravitational waves if it is misaligned with the rotation axis. The millisecond pulsar SAX J1808.4-3658 is an ideal system for testing this hypothesis, as discussed below. 


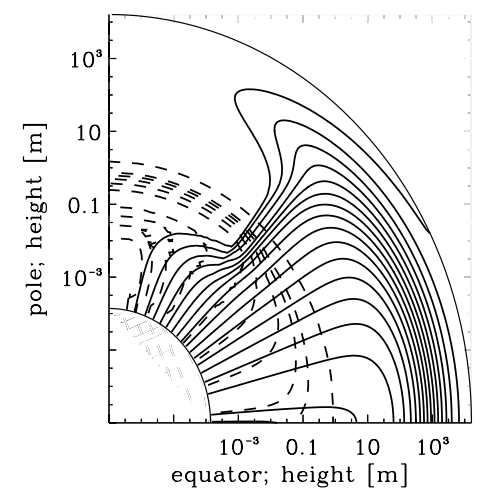

FIGURE 1. Equilibrium magnetic field lines (solid curves) and density contours (dashed curves) for $M_{\mathrm{a}}=10^{-5} M_{\odot}$, calculated numerically. The coordinates measure altitude above the stellar surface.

\section{MAGNETIC FIELD BURIAL}

\section{Hydromagnetic equilibria}

The steady-state, ideal-MHD equations for an isothermal atmosphere $\left(p=c_{\mathrm{s}}^{2} \rho\right)$ reduce to the force balance equation (SI units)

$$
\nabla p+\rho \nabla \phi-\mu_{0}^{-1}(\nabla \times \mathbf{B}) \times \mathbf{B}=0,
$$

where $\mathbf{B}$ denotes magnetic field, $\rho$ mass density, $p$ pressure, and $\phi$ gravitational potential. For an axisymmetric configuration we may write $\mathbf{B}=\nabla \psi(r, \theta) /(r \sin \theta) \times \hat{\mathbf{e}}_{\phi}$, leading to the Grad-Shafranov equation (Payne \& Melatos 2002)

$$
\Delta^{2} \psi=F^{\prime}(\psi) \exp \left[-\left(\phi-\phi_{0}\right) / c_{\mathrm{s}}^{2}\right]
$$

$F(\psi)=p(\psi) \exp \left[\left(\phi-\phi_{0}\right) / c_{\mathrm{s}}^{2}\right]$ traces the pressure along a flux tube outwards from the stellar surface, $\phi_{0}$ is the surface gravitational potential, and $\Delta^{2}$ is the Grad-Shafranov operator (Payne \& Melatos 2002). The hydromagnetic length-scale $|\mathbf{B}| /|\nabla \mathbf{B}|$ is much smaller than the hydrostatic length-scale $|p| /|\rho \nabla \phi|$, so that $\phi=G M_{*} r / R_{*}^{2}$. To close the problem, and connect the initial and final states uniquely, we require mass to be conserved in flux tubes in ideal MHD, according to:

$$
\frac{d M}{d \psi}=2 \pi \int_{C} \rho(r, \theta) \frac{r \sin \theta}{|\nabla \psi|} d s .
$$

$C$ is a magnetic field line, and the mass-flux distribution $d M / d \psi$ is prescribed.

With $\psi=0$ at the pole and $\psi=\psi_{*}$ at the equator, the total accreted mass $M_{\mathrm{a}}$ is assumed to be distributed as $d M / d \psi=\left(M_{\mathrm{a}} / 2 \psi_{\mathrm{a}}\right) \exp \left(-\psi / \psi_{\mathrm{a}}\right)$, where $\psi_{\mathrm{a}}=\psi_{*} R_{*} / R_{\mathrm{a}}$ 
is the flux enclosed by the inner edge of the accretion disk at a distance $R_{\mathrm{a}}$. For the boundary conditions, we fix $\psi$ to be dipolar at $r=R_{*}$, assume north-south symmetry, fix the $\psi=0$ field line and leave the field free at large $r$. The line-tying condition at $r=R_{*}$ does not allow any sinking of accreted material and is only valid when $M_{\mathrm{a}} \ll M_{\odot}$.

Equations (2) and (3) are solved numerically using an iterative relaxation scheme [5,6]. Convergence to the final state occurs monotonically for $M_{\mathrm{a}} \leq 10^{-5} M_{\odot}$ but is more erratic for larger $M_{\mathrm{a}}$. We also solve (2) and (3) analytically by a Green function method for the sake of verification [5]. Figure 1 shows an equilibrium configuration for $M_{\mathrm{a}} \leq$ $10^{-5} M_{\odot}$ and $\psi_{\mathrm{a}} / \psi *=10^{-1}$. Evident is the polar mountain, traced out by the dashed contours and the pinched, flaring magnetic field or 'tutu' [4]. The fractional deformation is given by the ellipticity $\varepsilon=2 \pi I_{0}^{-1} \int_{0}^{1} d(\cos \theta) \int_{R_{*}}^{\infty} d r r^{4}\left(3 \cos ^{2} \theta-1\right) \rho(r, \theta)$.

\section{Ohmic dissipation}

Ohmic dissipation allows the compressed equatorial magnetic field to relax by diffusing through the mountain, counteracting pile-up due to accretion. The Ohmic $\left(\tau_{\mathrm{d}}\right)$ and accretion $\left(\tau_{\mathrm{a}}\right)$ time-scales have been studied in one-dimensional geometry with the magnetic field parallel to the surface of the star [7, 8]. Growth of the polar mountain halts when $\tau_{\mathrm{a}}=M_{\mathrm{a}} / \dot{M}_{\mathrm{a}} \sim \tau_{\mathrm{d}}=\mu_{0} \sigma L^{2}$, where $\sigma$ is the electrical conductivity and $L=(|\psi| /|\nabla \psi|)_{\text {min }}$ is the characteristic length scale of the steepest field gradients [8]. This limits $\varepsilon$ to its value at $\tau_{\mathrm{a}} \approx \tau_{\mathrm{d}}$ (mass $M_{\mathrm{d}}$ ) [5]. We ignore the Hall effect and thermomagnetic drift in this work.

\section{MASS QUADRUPOLE AND MAGNETIC DIPOLE MOMENTS}

Figure $2 \mathrm{a}$ shows $\varepsilon$ as a function of $M_{\mathrm{a}}$. The mass quadrupole moment is $2 \varepsilon I_{z z} / 3$, where $I_{z z} \approx 0.4 M_{*} R_{*}^{2}$ is the moment of inertia. We plot both analytic and numerical results. The analytic results follow from a Green function analysis [5], and are given by $\varepsilon=5 M_{\mathrm{a}} /\left(2 M_{*}\right)(1-3 / 2 b)\left[1+M_{\mathrm{a}} b^{2} /\left(8 M_{\mathrm{c}}\right)\right]^{-1}, \mu=\psi_{*} R_{*}\left(1+M_{\mathrm{a}} / M_{\mathrm{c}}\right)^{-1}, M_{\mathrm{c}}=$ $\min \left[M_{\mathrm{d}}, 2 \pi G M_{*} \psi_{*}^{2} /\left(\mu_{0} R_{*}^{2} c_{\mathrm{s}}^{4}\right)\right]$, and $b=\psi_{*} / \psi_{\mathrm{a}}$. Numerical results are plotted for $b=3$, where convergence is most secure; they show good agreement.

$\varepsilon$ increases linearly with $M_{\mathrm{a}}$ until either (i) sufficient mass builds up in the polar mountain and causes it to spread towards the equator, limiting the growth of the polar deformation, or (ii) ohmic diffusion allows matter to spread across field lines. At larger $M_{\mathrm{a}}$, the saturation value $\varepsilon \sim 20\left(M_{\mathrm{c}} / M_{*}\right) b^{-2}$ depends only on the polar flux tube. For realistic accretion geometries, $b \sim 100$ gives $\varepsilon \sim 10^{-7}$, potentially leading to detectable levels of gravitational radiation.

The characteristic gravitational wave strain, averaged over polarization and orientation, is given by $h_{\mathrm{c}} \propto I_{z z} f^{2} \varepsilon / d$, where $d$ is the distance to the source [1]. Figure $2 \mathrm{~b}$ compares $h_{\mathrm{c}}$ with sensitivities of the initial and advanced detectors in LIGO for a coherent integration with $\tau=1 \mathrm{yr}$. Detections are expected for $M_{\mathrm{a}} \geq 10^{-5} M_{\odot}, f \geq 0.2 \mathrm{kHz}$, (LIGO), and $M_{\mathrm{a}} \geq 10^{-6} M_{\odot} f \geq 0.1 \mathrm{kHz}$ (advanced LIGO). 

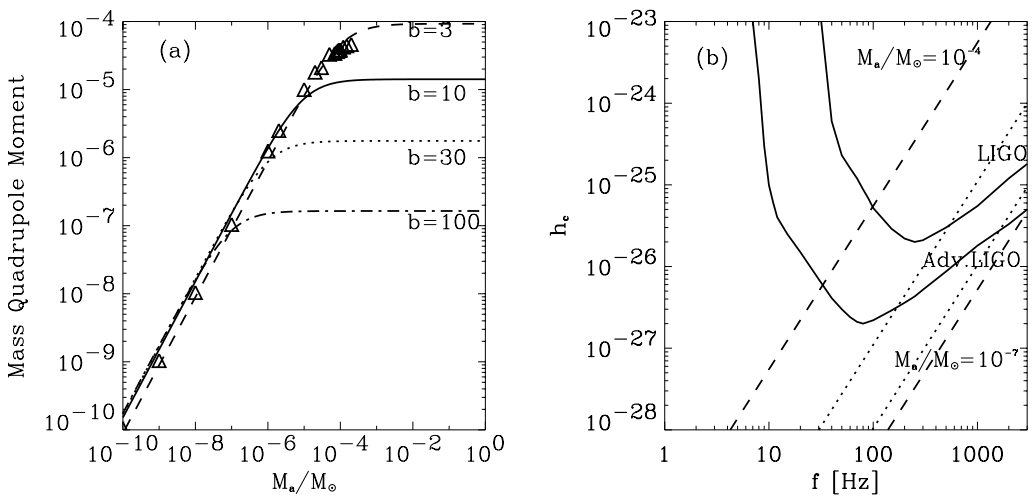

FIGURE 2. (a) Mass ellipticity as a function of $M_{\mathrm{a}}$ plotted for several $b$ values, with numerical results shown for $b=3$ for comparison. (b) Characteristic wave strain $h_{\mathrm{c}}$ as a function of known $f$ showing the weakest $h_{\mathrm{c}}$ detectable with $99 \%$ confidence in $\tau \sim 10^{7}$ s for LIGO and advanced LIGO, compared with the expected signal strength for a neutron star at $d=10 \mathrm{kpc}$. Lines are shown for $M_{\mathrm{a}} / M_{\odot}=10^{-7}, 10^{-4}$ with $b=30$ (dashed) and $b=3$ (dotted).

\section{DISCUSSION}

The mechanism of magnetic burial implies that millisecond X-ray pulsar periods such as SAX J1808.4-3658 may be detectable as gravitational wave sources with current generation interferometers. The signal strength is comparable to that invoked by Bildsten [2] to explain the narrow range of X-ray millisecond pulsars.

These sources have the advantage of persistence at a known frequency. For a neutron star accreting at the Eddington rate $\dot{M}_{\mathrm{a}}=10^{-8} M_{\odot} \mathrm{yr}^{-1}$, it takes only $10^{4} \mathrm{yr}$ to accrete enough mass to induce detectable gravitational radiation. Moreover, magnetic burial reduces the magnetic dipole moment as $\varepsilon$ increases, $[4,5]$, implying a scaling between $h_{\mathrm{c}}$ and $\mu$ that is observationally testable [9].

\section{REFERENCES}

1. Brady, P. R., and Creighton, T., Phys. Rev. D, 61, 82001 (2000).

2. Bildsten, L., ApJ, 501, L89 (1998).

3. Bonazzola, S., and Gourgoulhon, E., $A \&$ \& A, 312, 675 (1996).

4. Melatos, A., and Phinney, E. S., PASA, 18, 421 (2001).

5. Payne, D. J. B., and Melatos, A., submitted to MNRAS (2003).

6. Mouschovias, T. C., ApJ, 192, 37 (1974).

7. Brown, E. F., and Bildsten, L., ApJ, 496, 915 (1998).

8. Cumming, A., Zweibel, E., and Bildsten, L., ApJ, 557, 958 (2001).

9. Melatos, A., Payne, D. J. B., and Phinney, E. S., submitted to ApJ (2003). 